\title{
Indicators of reproductive performance in rainbow trout Oncorhynchus mykiss (Walbaum) selected for high and low responsiveness to stress
}

T G Pottinger \& T R Carrick

NERC Institute of Freshwater Ecology, Windermere Laboratory, The Ferry House, Far Sawrey, Ambleside, Cumbria LA22 0LP, United Kingdom

Correspondence: Tom Pottinger, NERC Institute of Freshwater Ecology, Windermere Laboratory, The Ferry House, Far Sawrey, Ambleside, Cumbria LA22 OLP

Tel.: 015394-42468

Fax: 015394-46914

Email: t.pottinger@ife.ac.uk

Running head: Stress responsiveness and reproduction in trout

Key words: stress, rainbow trout, reproduction, selective breeding, cortisol, estradiol-17 $\beta$ 


\section{Abstract}

A number of indicators of reproductive performance were assessed in rainbow trout divergently selected on the basis of their plasma cortisol response to a standardised stressor. For both male and female rainbow trout, body weight was significantly related to stress responsiveness. High-responding (HR) females were significantly heavier than lowresponding (LR) females overall and HR males were significantly heavier than LR males overall. There was no significant difference in the SGR of HR and LR female fish during the experimental period while in contrast the mean SGR of HR males was significantly greater than that of LR males. The divergent confinement-induced levels of blood cortisol in HR and LR groups were stable for more than one year after selection. There was no significant difference in the extent to which confinement stress reduced blood estradiol-17 $\beta$ levels in HR and LR females despite the large difference in relative blood cortisol levels. The onset and rate of ovulation was similar within the HR and LR groups. Differences in fecundity, relative fecundity and egg volume and weight were wholly attributable to the different mean body weights of the HR and LR fish. There was no difference between the sperm counts of HR and LR males. There was significantly higher mortality among eggs derived from HR parents overall between fertilization and hatch but maximum mean mortality did not exceed $12 \%$. Mean time to eyeing in the HR groups was 224 degree days and in the LR groups was 244 degree days. Hatching commenced at 344 and 347 degree days in the HR and LR groups respectively and was complete within 412 and 416 degree days respectively. Overall, selection for stress responsiveness was associated with no significant benefits or costs in reproductive performance. 


\section{Introduction}

Because husbandry practices are of necessity compromised by the economic realities of large-scale fish production, stress is an unavoidable component of the finfish aquaculture environment (Barton, 1997). Although the physiological response of fish to an isolated stressor is essentially adaptive, fish under intensive culture conditions are exposed to a regime of repeated acute stress (handling, grading, transport, prophylactic treatment) and in some instances chronic stress (deterioration in water quality, overcrowding, social interaction).

Frequent or prolonged activation of the stress response of fish can have significant adverse effects on growth (Pickering, 1993; Pankhurst \& van der Kraak, 1997), reproductive function (Pickering, Pottinger, Carragher \& Sumpter, 1987; Campbell, Pottinger \& Sumpter, 1992, 1994) and immunocompetence (Balm, 1997). In addition, stress-induced biochemical changes in muscle prior to slaughter have been demonstrated to have deleterious effects on the flesh quality of fish (Lowe, Ryder, Carragher \& Wells , 1993; Sigholt, Erikson, Rustad, Johansen, Nordtvedt \& Seland, 1997). Therefore, it has been suggested that within an environment in which stressful stimuli are frequent and/or prolonged, fish which are less responsive to stressful stimuli will perform better than fish displaying a more pronounced sensitivity to stress (Pottinger \& Pickering, 1997).

Considerable effort has been devoted to defining the heritability of economically important traits in fish, such as growth rate, size at maturity and flesh quality (see Tave, 1987), and 
several studies published in recent years suggest that selective breeding of finfish for reduced responsiveness to stress is possible. The level of the steroid hormone cortisol in the blood is a major index of stress in fish (Barton \& Iwama, 1991), and cortisol is a causal factor in many of the deleterious effects of stress (Pickering \& Pottinger, 1989; Pankhurst \& van der Kraak, 1997). Thus post-stress cortisol levels provide a trait of functional significance upon which selection pressure can be directed. The magnitude of the cortisol response to stress of individual rainbow trout (Oncorhynchus mykiss) is stable over time (Pottinger, Pickering \& Hurley, 1992) and both semi-quantitative (Pottinger, Moran \& Morgan, 1994) and quantitative (Fevolden, Roed, Fjalestad \& Stein, 1999; Pottinger \& Carrick, 1999a) studies have demonstrated that the magnitude of the cortisol response to stress is heritable in rainbow trout. These results suggest that modification of the endocrine stress response in fish, with the attendant benefits this would bring, is technically feasible.

It is presently unclear, however, whether modification of the stress response is of significance to the fish other than under stressful conditions or whether selection for stress responsiveness inadvertently co-selects additional undesirable traits. The aim of this study was to assess the variation in a range of indicators of reproductive performance in fish selected on the basis of their responsiveness to a standardized stressor, under relatively benign, minimal stress, conditions. 


\section{Materials and methods}

\section{Fish husbandry}

During February 1996, two hundred and fifty two-year old mixed sex rainbow trout (Stirling strain) were divided evenly between ten 15001 holding tanks, each supplied with a constant flow of lake water $\left(251 \mathrm{~min}^{-1}\right)$. As far as was possible the fish were segregated on the basis of sex, after external examination. Six tanks of female fish and four tanks of male fish resulted (25 fish/tank). Each fish was weighed, measured, and individually tagged with a passive integrated transponder (PIT) tag (Fish Eagle Co.) which was inserted into the peritoneal cavity of each fish. On subsequent occasions, PIT tags were implanted into the dorsal musculature because of tag loss associated with the ejection of eggs from the body cavity in ripe females.

\section{Evaluation of individual stress responsiveness}

One month after the fish were distributed and tagged, and at monthly intervals following, the fish from each holding tank were transferred, in turn, to four 501 confinement tanks, $6 / 7$ fish per tank. Each confinement tank was supplied with a constant flow of lake water (15 1 $\left.\min ^{-1}\right)$. After $3 \mathrm{~h}$ confinement, the fish from the first confinement tank were transferred to anaesthetic (2-phenoxyethanol, 1:2000) and a blood sample $(<1.0 \mathrm{ml})$ was removed from the cuverian sinus of each fish into a heparinized syringe. After blood sampling, the fish was identified by tag number, and weight and length were recorded before the fish was returned to its original holding tank to recover. The blood samples were kept on ice until being centrifuged. The resultant plasma was stored frozen $\left(-20^{\circ} \mathrm{C}\right)$ until required for assay. 
For each sample coefficient of condition (K-factor; 100.weight/length ${ }^{3}$ ) and specific growth rate $\left(\mathrm{SGR}\right.$; where SGR $=\left[\left(\ln W_{2}-\ln W_{1}\right) /\left(t_{2}-t_{1}\right)\right] .100$ and $W_{1}$ and $W_{2}$ are body weight at the start and end of the growth period, and $t_{2}-t_{1}$ is the length of the period in days) were calculated.

It was intended to complete the selection procedure within 6 months. However, during the period February to May, considerable problems were encountered with a virulent outbreak of fungal disease (Saprolegnia spp.). The pathogen did not respond satisfactorily to the normal therapeutic procedure (flush treatment with malachite green) and $80 \%$ of the male fish died as a result of the fungal infection or were killed to alleviate suffering [Fungal infection is frequently associated with sexual maturity in male salmonids (Pickering and Christie, 1980)]. Consequently, an additional group of 100 rainbow trout of the same age (Stannan strain) were recruited. It was not possible to establish the sex of these fish because no external secondary sexual characters are discernible in rainbow trout during the interspawning period. The fish were PIT-tagged and distributed randomly between four 15001 tanks, 25 fish per tank. These fish were subjected to the same regime of periodic confinement stress and blood sampling as the original group of fish on four occasions during the period June to October.

\section{Identification and segregation of high- and low-responding fish}

When all monthly samples were completed and assayed, the mean post-stress plasma cortisol level for each fish was calculated and the fish within each tank were ranked on the basis of this figure. Fish which were ranked in the top four in each tank were designated 
high-responders (HR) and those ranked in the lowest four were denoted low-responders (LR). The fish selected as HR and LR were removed from their holding tanks and segregated in tanks containing only male HR or LR or female HR or LR. At this stage each group comprised 24 fish.

\section{Execution of crosses}

During January and February 1997, all selected female fish were examined at regular intervals to assess whether ovulation had yet occurred. Ovulated females were anaesthetised individually and the date of ovulation was recorded, together with weight, length and PIT tag number. The eggs were expressed from the body cavity by gentle pressure on the abdomen. Eggs were collected in a mesh-bottomed plastic bowl and ovarian fluid was drained before the total egg weight was determined. Ten eggs were retained on ice in a capped plastic tube for measurement of egg diameter, using a dissection binocular microscope and eyepiece graticule, and individual weight. The spent fish was then returned to a holding tank separate from those containing fish not yet ovulated. The eggs were fertilised using milt stripped from a mature male chosen randomly from the corresponding selection group (HR or LR). The male was anaesthetised, weighed, measured and the PIT tag number was recorded. Approximately $5 \mathrm{ml}$ of milt were collected into a plastic tube which was placed on ice. The male was returned to a separate holding tank. Approximately $1.0 \mathrm{ml}$ of milt was used to fertilise 1000 eggs, with the assumption being made that each female was expressing approximately 2500 eggs. Gentle mixing of the eggs and milt was carried out dry in a plastic bowl before a small amount of water was added and further mixed. The eggs derived from each cross were then transferred to individual mesh baskets 
in an egg incubator trough supplied with a constant flow of lake water. Eggs were monitored through to hatch and mortalities were recorded. Total numbers of eggs in each family were determined at the eyed egg stage. No distinction was made between fertile and infertile eggs when recording losses.

A $10 \mu \mathrm{l}$ aliquot of the remaining milt was added to $10.0 \mathrm{ml}$ of $5 \%$ saline in a plastic tube and mixed well. The sperm were counted using a haemocytometer at 250x magnification, enumerating 10 fields of view and calculating the concentration of sperm per $\mu 1$ of milt accordingly. Gonadosomatic index was calculated for the females as [(total egg mass/total body weight) x100]. Mean egg volume was calculated from the measurement of egg diameter $(n=10)$, and the total number of eggs and number of eggs $/ \mathrm{kg}$ body weight were calculated from individual egg weight $(\mathrm{n}=10)$, total egg mass, and stripped body weight.

\section{The effect of confinement stress on blood cortisol and estradiol-17 $\beta$ levels in HR and}

\section{LR parent fish}

Ten HR and ten LR fish, chosen randomly from the survivors of the spawning exercise, were maintained in two tanks following spawning. All fish were female because of the very low survivorship among the mature male fish following spawning. The fish were fed three times weekly at the manufacturers recommended rate (Trouw Standard Expanded 60). In November 1997, the fish were transferred from their holding tanks, anaesthetised, blood sampled, weighed and measured, identified by PIT tag, and transferred to confinement tanks (50 1), three fish per confinement tank. Further blood samples were taken from each fish at intervals of $2,3,4,5,7,24,48$, and $168 \mathrm{~h}$ after transfer to the confinement tanks. 
Plasma cortisol and estradiol-17 $\beta$ (E2) levels were measured to assess whether the degree of disruption to the pituitary-gonadal axis caused by confinement stress varied between the two selected groups.

\section{Assay procedures}

Plasma cortisol and E2 levels were determined by validated radioimmunoassay procedures (Pickering, Pottinger \& Sumpter, 1987; Pottinger \& Pickering, 1990).

\section{Statistical analysis}

Multifactorial analysis of variance (ANOVA, Genstat 5, Lawes Agricultural Trust) was employed to assess the significance of changes with time and differences within and between groups in length, weight, coefficient of condition, specific growth rate, and plasma steroid levels. Differences in reproductive characteristics were also assessed by ANOVA. For the analysis of fecundity and egg size data, body weight was included as a covariate, with the analyses being carried out on log-transformed data sets. Egg mortality data were also analysed by ANOVA after being converted to proportions and arcsin-transformed.

\section{Results}

\section{Weight, length, condition and growth rate of HR and LR fish}

For both male and female rainbow trout, body weight was significantly related to stress responsiveness. The mean weight of HR females during the entire period of the study was 
significantly greater than that of LR females $(P<0.001 ; 544 \pm 12$ c.f. $405 \pm 9 \mathrm{~g})$ and, similarly, HR males were significantly heavier, overall, than LR males $(P<0.001 ; 696 \pm 22$ c.f. $579 \pm 22$ g). The significant difference in weight between the female HR and LR groups was sustained throughout the sampling period (Fig. 1) but the difference in weight between male HR and LR groups was significant only from the third sample onwards (Fig. 1). Fork length also varied significantly between groups; HR fish were larger than LR fish $(P<0.001$; females $34.2 \pm 0.2$ c.f. $31.7 \pm 0.2 \mathrm{~cm}$; males $36.3 \pm 0.3$ c.f. $34.0 \pm 0.3 \mathrm{~cm})$. There was no significant difference in K-factor between HR and LR males $(1.416 \pm 0.02$ c.f. $1.406 \pm 0.013$ ) but female HR K-factor was significantly greater than female LR K-factor $(P<0.05 ; 1.333 \pm 0.012$ c.f. $1.254 \pm 0.011)$. There was no significant difference in the SGR of HR and LR female fish during the experimental period ( $0.351 \pm 0.03$ c.f. $0.312 \pm 0.04 \%$ body weight day ${ }^{-1}$ ) while in contrast the mean SGR of HR males was significantly greater than that of LR males $\left(P<0.01 ; 0.506 \pm 0.04\right.$ c.f. $0.371 \pm 0.04 \%$ body weight day $\left.{ }^{-1}\right)$.

At spawning (January/February) female HR fish remained significantly larger than LR fish (Table 1). However, among males the situation had reversed and LR fish were now larger $(P=0.06)$ than HR males (Table 2$)$.

\section{Plasma cortisol levels in HR and LR fish}

The relative divergence in confinement-induced plasma cortisol levels between HR and LR groups remained similar for both males and females throughout the testing period with a two- to three-fold increment between LR and HR levels. These data are reported fully 
elsewhere (Pottinger \& Carrick, 1999b).

\section{The effect of confinement stress on plasma cortisol and E2 levels in HR and LR fish}

More than 12 months after the initial selection procedure, surviving HR and LR female fish still displayed divergent body weights (LR: $2433 \pm 139 \mathrm{~g} ;$ HR $3091 \pm 174 \mathrm{~g} ; P<0.01)$ and cortisol response to confinement (Fig. 2a). Levels in LR fish were significantly elevated from a baseline of $59.8 \pm 9 \mathrm{ng} \mathrm{ml}^{-1}$ to $208 \pm 22 \mathrm{ng} \mathrm{ml}^{-1}$ within $2 \mathrm{~h}$ of the onset of confinement $(P<0.001)$. In contrast, levels in HR fish increased from a similar baseline of $45 \pm 9 \mathrm{ng} \mathrm{ml}^{-1}$ to reach $342 \mathrm{ng} \mathrm{ml}^{-1}$ within $2 \mathrm{~h}(P<0.001)$. Levels in the HR fish were significantly higher than those in the LR fish at all subsequent time points until $48 \mathrm{~h}$ at where the difference was not significant. In both HR and LR fish cortisol levels remained significantly $(P<0.001)$ higher than those at time 0 until at least $48 \mathrm{~h}$ after the onset of confinement. During the period $2 \mathrm{~h}$ to $48 \mathrm{~h}$ there was no significant decline in cortisol levels in the LR fish. In the HR fish, however, levels declined significantly during this period $(P<0.05)$. Within $168 \mathrm{~h}$ levels returned to baseline in both groups.

There was no significant difference in blood E2 levels between HR and LR fish at any point during the confinement period $(P=0.135$; Fig. $2 b)$. However, there was a significant decline in E2 levels in both groups between 2 and $24 \mathrm{~h}$ after the onset of confinement $(P<0.01)$ and subsequently a significant increase in E2 levels between 24 and $168 \mathrm{~h}$. E2 levels at $336 \mathrm{~h}$ were statistically indistinguishable from those at $0 \mathrm{~h}$. 


\section{Reproductive characteristics of HR and LR fish}

Among the females, ovulation occurred over a period of 45 days from the time at which the first fish to ovulate was detected. The rate of ovulation was similar within the HR and LR groups (Fig 3).

The data obtained from mature female HR and LR fish at the time of stripping are presented in Table 1. As was the case during the selection period 6 months previously, HR fish were significantly larger than LR fish. However, there was no significant difference in GSI between HR and LR fish. Neither fecundity nor egg size were significantly different between HR and LR groups when body weight (post-stripping) was included as a covariate in the ANOVA. There was no difference between the sperm counts of HR and LR males (Table 2).

Analysis of the arcsin-transformed egg mortality data revealed there to be a significant difference $(P<0.001)$ in mortality levels between progeny groups derived from HR or LR parents overall between fertilization and hatch (HR: $12.5 \pm 1.8 \%$; LR: $8.0 \pm 2.2 \%$; Fig. 4). Mean time to eyeing in the HR groups was 224 degree days and in the LR groups was 244 degree days. Hatching commenced at 344 and 347 degree days in the HR and LR groups respectively and was complete within 412 and 416 degree days respectively. 


\section{Discussion}

These data suggest that, in the absence of adverse environmental factors, the selection of fish on the basis of stress responsiveness does not significantly affect reproductive performance. Differences in fecundity and egg size which were evident between highresponding (HR) and low-responding (LR) fish can be accounted for by the fact that HR females were significantly larger fish than LR females. These results raise a number of points which are considered in more detail below.

The population of rainbow trout used for this study contained subsets with consistently high and low responsiveness to stress, measured as plasma cortisol elevation in response to confinement (Pottinger \& Carrick, 1999a). Stability of individual stress responsiveness over time would be expected based on previous results (Pottinger, Pickering \& Hurley, 1992) and in the present study the divergence in cortisol response to confinement of the selected female fish was still evident 10 months after spawning (Fig. 2).

There was a positive correlation between cortisol responsiveness and size in fish selected from this population on the basis of stress responsiveness, with HR fish being larger than LR fish. This counter-intuitive relationship between size and stress-responsiveness has previously been reported for female fish (Pottinger \& Carrick, 1999b) but these data show that it is also evident in male fish. The significant difference in weight between HR and LR females was apparent from the start of testing (March) through to spawning (January/February of the following year) but in male fish the difference in weight between 
HR and LR individuals was not apparent until August and was reflected in a significantly different SGR for the two groups during the testing period. The reasons underlying the overall larger size of HR fish remain to be established and it should be noted that the progeny of the HR and LR parents do not show this trend (T. G. Pottinger \& T. R. Carrick, unpublished) suggesting underlying environmental rather than genetic causes. The factors which determine size and growth in captive fish are complex (see Cutts, Metcalfe \& Taylor, 1998, for discussion) but reside in part in the relative success of individual fish in acquiring food. It is possible that (yet to be defined) behavioural traits associated with stress responsiveness interact with the environment to affect the success of individuals in competitive interaction for food.

At spawning time, LR males were larger than HR males, reversing the trend observed during the selection period for HR fish to be larger than LR fish. During selection (June October) the male fish were maintained in mixed-sex, mixed-responsiveness populations, but following the identification of HR and LR individuals these were transferred to tanks containing only HR or LR fish of the same sex. During the pre-spawning period male salmonid fish in captivity exhibit aggression and territoriality and engage in agonistic interactions. Furthermore, blood cortisol levels are elevated in salmonid fish of both sexes during the pre-spawning months (Pickering \& Christie, 1981). This is evident in the high levels of cortisol apparent in the mature female fish prior to confinement in the present study (approx. $50 \mathrm{ng} \mathrm{ml}^{-1}$; Fig. 2). It is possible that a combination of these factors affected growth in the male HR fish more markedly than in the LR fish, because of the tendency of HR fish to produce higher blood cortisol levels in response to stress. If this is the case, the 
benefits or otherwise of possessing an enhanced responsiveness to stressors may vary with sex and developmental stage.

Interest in the aquacultural value of fish with reduced responsiveness to stress resides in the assumption that their performance under adverse conditions will be enhanced relative to fish which display a more pronounced responsiveness to stress. It should be emphasised that the present study was designed to assess whether there were underlying differences in reproductive performance between HR and LR fish, independent of adverse environmental factors. The imposition of stress during the reproductive period is known to have negative effects in a number of species (see Pankhurst \& van der Kraak, 1997) including reduced progeny survival (Campbell, Pottinger \& Sumpter, 1992). Because the progeny from these crosses were required for further work the fish were not submitted to any unnecessary disturbance during the course of this study. Thus, these data do not indicate how the imposition of additional stress might affect relative performance of HR and LR fish.

In the male fish there was no significant difference in sperm count between HR and LR fish at spawning. Motility of the sperm and fertilizability of the sperm were not assessed directly. Each male fertilized eggs from a single female so overall egg survival is a function of both male and female contributions. The ova were not shocked after eyeing to identify unfertilised eggs and overall egg mortality levels therefore include both fertilised and unfertilised eggs. The significantly higher losses among the HR ova suggests that there may have been a difference in either, or both, the quality of sperm or eggs between selection groups. Milt quality is generally defined as the ability to fertilize eggs (Aas, Refstie \& 
Gjerde, 1991) and high quality semen is suggested to provide a fertilization rate $>80 \%$ (Lahnsteiner, Berger, Weismann \& Patzner, 1998). Factors contributing to egg quality are ill-defined (Brooks, Tyler \& Sumpter, 1997). However, despite the statistically significant difference in egg mortality between selection groups the mean losses of ova among the HR progeny groups did not exceed $12 \%$ suggesting a fertilization rate of at least $88 \%$ had been achieved and indicating that milt from both HR and LR males was of high quality.

Among the mature females, the difference in body weight between HR and LR fish which was apparent during the selection process was still evident at spawning. HR and LR fish ovulated over the same period of time, and at the same rate. Previous reports suggest that body weight before sexual maturity, and time of spawning within a season, are not related (Crandell \& Gall, 1993). Generally, fecundity, egg size and egg volume in rainbow trout are reported to increase with fish size (Bromage \& Cumaranatunga, 1988) and therefore these data sets were analysed with body weight as a covariate to compensate for the different mean body weights of the HR and LR groups. When stripped body weight was taken into account there were no significant differences between HR and LR females in the total number of eggs ovulated (fecundity), eggs per unit body weight (relative fecundity), or individual egg weight and individual egg volume.

Arguably, larger eggs might be assumed to possess larger reserves of yolk and therefore offer an advantage to the developing embryo/alevin. The apparent differences in individual egg weight and volume observed in the HR and LR groups is fully accounted for by the difference in body weight of these fish but if HR females are inherently larger than LR 
females this might be construed as a potential advantage. The egg mortality pattern did not support this contention. Previous reports indicate that there is no relationship between fertilization rate and egg size (Bromage \& Cumaranatunga, 1988) or mortality and egg size (Bromage \& Cumaranatunga, 1988) and that although larger eggs produce larger fry, this advantage is insignificant compared to other factors affecting growth size (Bromage, Jones, Randall, Thrush, Davies, Springate, Duston \& Barker, 1992).

The HR and LR groups of fish were not subjected to any additional stressors during the period prior to spawning for reasons discussed above. However, during the following year's reproductive cycle, mature female fish from both HR and LR groups were subjected to a prolonged period of confinement in order to assess the effects of chronic stress on gonadal steroid production, as evidenced by plasma estradiol-17 $\beta$ (E2) levels. Stress, and elevated blood cortisol, have been shown to suppress circulating E2 levels (Pankhurst \& van der Kraak, 1997). It was therefore assumed that if the possession of either HR or LR trait impacted differentially upon the reproductive endocrine system during stress, this would be reflected in a differential modulation of gonadal steroid levels.

During a two week period of continuous confinement the difference in blood cortisol levels between HR and LR fish was sustained for between 24 and $48 \mathrm{~h}$. This indicates both that sexual maturation does not abolish the difference in responsiveness between the selected groups and that the divergence in blood cortisol levels arises due to differences in the maximum stress-induced cortisol level achieved, not via a difference in dynamics of the response. This is consistent with the time-course of the response to confinement in the HR 
and LR progeny of these fish (Pottinger \& Carrick, 1999a). The present data also suggest that cortisol levels in the HR fish declined from maximum values more quickly than those in LR fish, consistent with previous results (Pottinger, Moran \& Morgan, 1994).

Although the confined HR and LR females displayed a very pronounced difference in plasma cortisol levels for at least a 24 - $48 \mathrm{~h}$ period, the reduction of E2 levels subsequent to this elevation of cortisol was of a similar magnitude in both HR and LR fish. Arguably, if the adverse effects of stress on reproductive function are to a large extent (though not exclusively) mediated by disruption of gonadal steroid function these data suggest that, over the short-term at least, the impact of stress on reproductive function would be similar in these groups of HR and LR fish.

In summary, two groups of rainbow trout with divergent cortisol responses to confinement were identified. Despite the robust nature of the divergent cortisol responses to confinement no evidence of systematic differences among indicators of reproductive performance was apparent in the HR fish of either sex maintained under undisturbed conditions. During chronic confinement there was no difference in the degree to which E2 levels were suppressed in HR and LR female fish despite significantly divergent cortisol responses. These data indicate that neither HR nor LR trait is associated with an adverse effect on reproductive performance under routine husbandry conditions and that under conditions of stress divergent cortisol responsiveness of the magnitude present in these HR and LR groups may not be reflected in divergent impacts upon the reproductive endocrine system. Selection of rainbow trout for stress responsiveness does not co-select any disadvantageous 
reproductive traits.

\section{Acknowledgements}

This study has been carried out with financial support from the Commission of the European Communities, Agriculture and Fisheries (FAIR) specific RTD programme CT95152 'Selective breeding for stress tolerance in aquacultured fish' and from the Natural Environment Research Council of the UK.

\section{References}

Aas, G. H., Refstie, T. \& Gjerde, B. (1991) Evaluation of milt quality of Atlantic salmon. Aquaculture 95, 125-132.

Balm P. H. M. (1997) Immune-endocrine interactions. In: Fish Stress and Health in Aquaculture (ed. by G. K. Iwama, A. D. Pickering, J. P. Sumpter \& C. B. Schreck), pp. 195-221. Cambridge University Press, Cambridge.

Barton B. A. (1997) Stress in finfish: past, present and future - a historical perspective. In: Fish Stress and Health in Aquaculture (ed. by G. K. Iwama, A. D. Pickering, J. P. Sumpter \& C. B. Schreck), pp. 1-33. Cambridge University Press, Cambridge. 
Barton B. A. \& Iwama G. K. (1991) Physiological changes in fish from stress in aquaculture with emphasis on the response and effects of corticosteroids. Annual Review of Fish Diseases 1, 3-26.

Bromage N. \& Cumaranatunga P. R. C. (1988) Egg production in the rainbow trout. In Recent Advances in Aquaculture, Vol. 3 (ed. by R. J. Roberts \& J. F. Muir), pp. 63138, Croom Helm, London.

Bromage N., Jones J., Randall C., Thrush M., Davies B., Springate J., Duston J. \& Barker G. (1992) Broodstock management, fecundity, egg quality and the timing of egg production in the rainbow trout (Oncorhynchus mykiss). Aquaculture 100, 141-166.

Brooks S., Tyler C. R. \& Sumpter J. P. (1997) Egg quality in fish: what makes a good egg? Reviews in Fish Biology and Fisheries 7, 387-416.

Campbell P. M., Pottinger T. G. \& Sumpter J. P. (1992) Stress reduces the quality of gametes produced by the rainbow trout. Biology of Reproduction 47, 1140-1150.

Campbell P. M., Pottinger T. G. \& Sumpter J. P. (1994) Preliminary evidence that chronic confinement stress reduces the quality of gametes produced by brown and rainbow trout. Aquaculture 120, 151-169.

Crandell P. A. \& Gall G. A. E. (1993) The genetics of age and weight at sexual maturity 
based on individually tagged rainbow trout (Oncorhynchus mykiss). Aquaculture 117, 95-105.

Cutts C. J., Metcalfe N. B. \& Taylor A. C. (1998) Aggression and growth depression in juvenile Atlantic salmon: the consequences of individual variation in standard metabolic rate. Journal of Fish Biology 52, 1026-1037.

Fevolden S.-E., Rped K. H., Fjalestad K. T. \& Stien J. (1999) Post-stress levels of lysozyme and cortisol in adult rainbow trout (Oncorhynchus mykiss); heritabilities and genetic correlations. Journal of Fish Biology 54, 900-910.

Lahnsteiner F., Berger B., Weismann T. \& Patzner R. A. (1998) determination of semen quality of the rainbow trout, Oncorhynchus mykiss, by sperm motility, seminal plasma parameters, and spermatozoal metabolism. Aquaculture 163, 163-181.

Lowe T. E., Ryder J. M., Carragher J. F. \& Wells R. M. G. (1993) Flesh quality in snapper, Pagrus auratus, affected by capture stress. Journal of Food Science 58, 770 - 773.

Pankhurst N. W. \& van der Kraak G. (1997) Effects of stress on reproduction and growth of fish. In: Fish Stress and Health in Aquaculture (ed. by G. K. Iwama, A. D. Pickering, J. P. Sumpter \& C. B. Schreck), pp. 73-93. Cambridge University Press, Cambridge. 
Pickering A. D. (1993) Growth and stress in fish production. Aquaculture 111, 51-63.

Pickering A. D. \& Christie P. (1980) Sexual differences in the incidence and severity of ectoparasitic infestation of the brown trout, Salmo trutta L. Journal of Fish Biology 16, 669-683.

Pickering A. D. \& Christie P. (1981) Changes in the concentrations of plasma cortisol and thyroxine during sexual maturation of the hatchery-reared brown trout, Salmo trutta L. General and Comparative Endocrinology 44, 487-496.

Pickering A. D. \& Pottinger T. G. (1989) Stress responses and disease resistance in salmonid fish: effects of chronic elevation of plasma cortisol. Fish Physiology and Biochemistry 7, 253-258.

Pickering A. D., Pottinger T. G., Carragher J. F. \& Sumpter J. P. (1987) The effects of acute and chronic stress on the levels of reproductive hormones in the plasma of mature male brown trout, Salmo trutta L. General and Comparative Endocrinology 68, 249-259.

Pottinger T. G. \& Carrick (1999a) Modification of the plasma cortisol response to stress in rainbow trout by selective breeding. General and Comparative Endocrinology (In press). 
Pottinger T. G. \& Carrick T. R. (1999b) A comparison of plasma glucose and plasma cortisol as selection markers for high and low stress-responsiveness in female rainbow trout. Aquaculture 175, 351-363.

Pottinger T. G. \& Pickering A. D. (1990) The effect of cortisol administration on hepatic and plasma estradiol-binding capacity in immature female rainbow trout (Oncorhynchus mykiss). General and Comparative Endocrinology 80, 264-273.

Pottinger T. G. \& Pickering A. D. (1997) Genetic basis to the stress response: selective breeding for stress-tolerant fish. In: Fish Stress and Health in Aquaculture (ed. by G. K. Iwama, A. D. Pickering, J. P. Sumpter \& C. B. Schreck), pp. 171-193. Cambridge University Press, Cambridge.

Pottinger T. G., Pickering A. D. \& Hurley M. A. (1992) Consistency in the stress response of individuals of two strains of rainbow trout, Oncorhynchus mykiss. Aquaculture 103, 275-289.

Pottinger T. G., Moran T. A. \& Morgan J. A. W. (1994) Primary and secondary indices of stress in the progeny of rainbow trout (Oncorhynchus mykiss) selected for high and low responsiveness to stress. Journal of Fish Biology 44, 149-163.

Sigholt T., Erikson U., Rustad T., Johansen S., Nordtvedt T. S. \& Seland A. (1997) Handling stress and storage temperature affect meat quality of farmed-raised 
Atlantic salmon (Salmo salar). Journal of Food Science 62, 898-905.

Tave D. (1987). Genetics for fish hatchery managers. AVI Publishing Co. Inc.: Connecticut. pp. 299. 
Table 1 Somatic and reproductive measurements in female rainbow trout selected for high (HR) or low (LR) plasma cortisol responsiveness to confinement. Each value is the mean \pm SEM, $\mathrm{n}=14(\mathrm{LR})$ or 24 (HR). $P$ values are derived from one-way ANOVA (weight, fork length) and ANCOVA (all others).

LR HR

\begin{tabular}{lccc}
\hline \multicolumn{1}{c}{ Parameter } & mean \pm SEM & mean \pm SEM & $P$ \\
\hline Body weight $(\mathrm{g})$ & $1246 \pm 60$ & $1538 \pm 61$ & 0.008 \\
Fork length $(\mathrm{cm})$ & $42.0 \pm 0.7$ & $44.0 \pm 0.5$ & 0.03 \\
GSI & $15.5 \pm 1.0$ & $14.4 \pm 0.6$ & 0.33 \\
Total no. eggs & & & 0.48 \\
Eggs kg ${ }^{-1}$ body weight* & $2540 \pm 165$ & $2776 \pm 179$ & 0.7 \\
& $2545 \pm 201$ & $2101 \pm 125$ & 0.3 \\
Individual egg weight $\left(\mathrm{mg}^{*}\right)$ & $75.3 \pm 2.2$ & $80.2 \pm 1.6$ & 0.1 \\
\hline Individual egg volume $\left(\mathrm{mm}^{3}\right)$ & $74.3 \pm 2.0$ & $80.8 \pm 9.3$ & \\
\hline
\end{tabular}

* body weight post-stripping 
Table 2 Mean length, weight and sperm counts in male rainbow trout selected for high (HR) or low (LR) plasma cortisol responseto confinement. Each value is the mean $\pm S E M$, n $=14$ (LR) or 24 (HR). Quoted $P$ values are derived from one-way ANOVA.

\begin{tabular}{cccc} 
& LR & HR & \\
\hline Parameter & mean \pm SEM & mean \pm SEM & $P$ \\
\hline Body weight $(\mathrm{g})$ & $1237 \pm 54$ & $1075 \pm 54$ & 0.06 \\
Fork length $(\mathrm{cm})$ & $42.6 \pm 0.6$ & $41.4 \pm 0.5$ & 0.14 \\
Sperm count $\left(10^{6} \mu \mathrm{l}^{-1}\right)$ & $14.8 \pm 1.3$ & & 0.5 \\
\hline
\end{tabular}




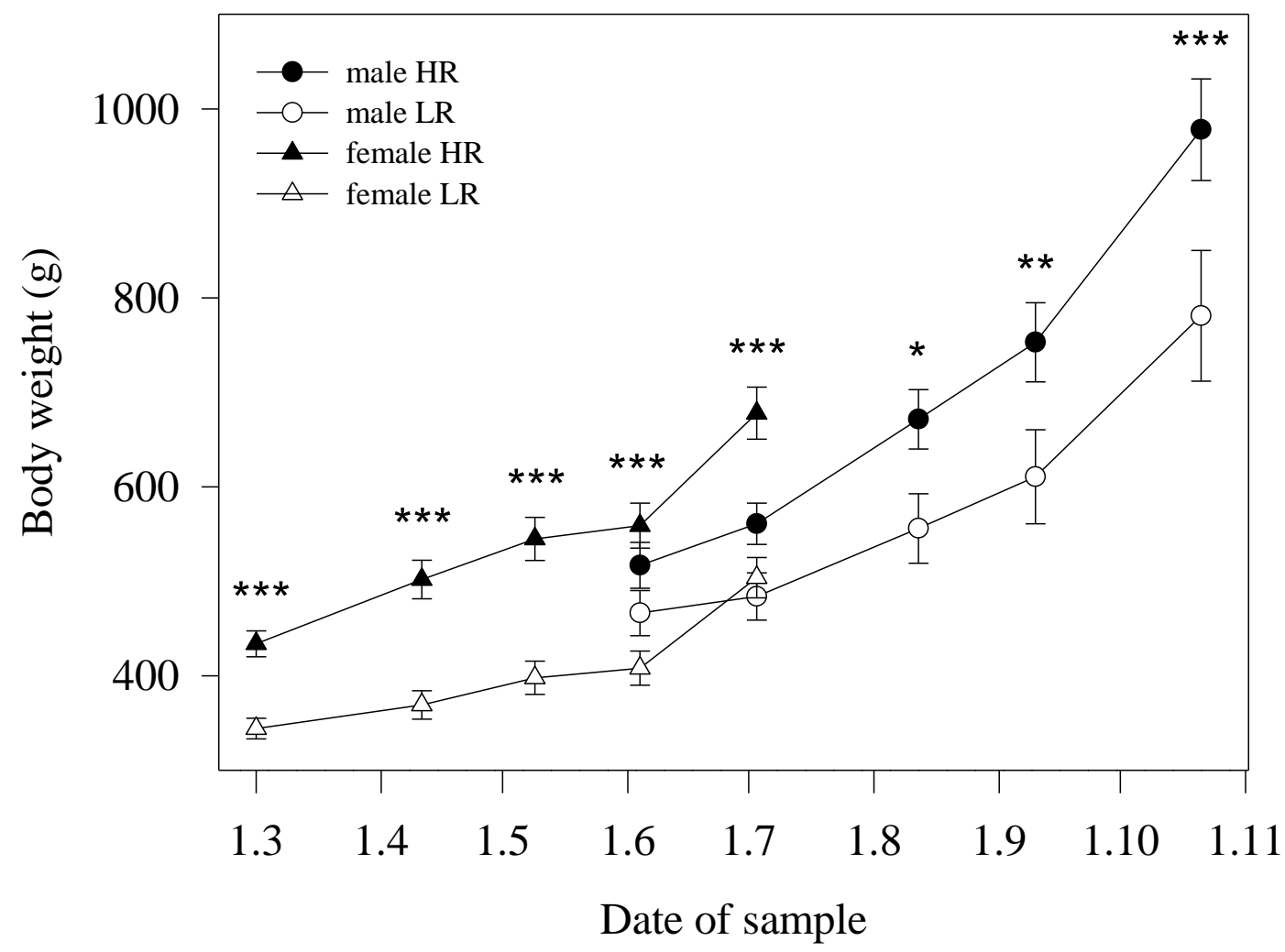

Figure 1 Changes in body weight with time of female $(\mathbf{\Delta})$ and male $(\mathbf{O})$ fish selected as high-responding (HR; solid) or low-responding (LR; open) on the basis of their plasma cortisol response to a confinement stressor. Female fish were tested during the period March - July while male fish were tested during the period June - October. Each point is the mean \pm SEM, $\mathrm{n}=23$. Significant differences between HR and LR fish at each time are denoted by $* P<0.05, * * P<0.01, * * * P<0.001$. 

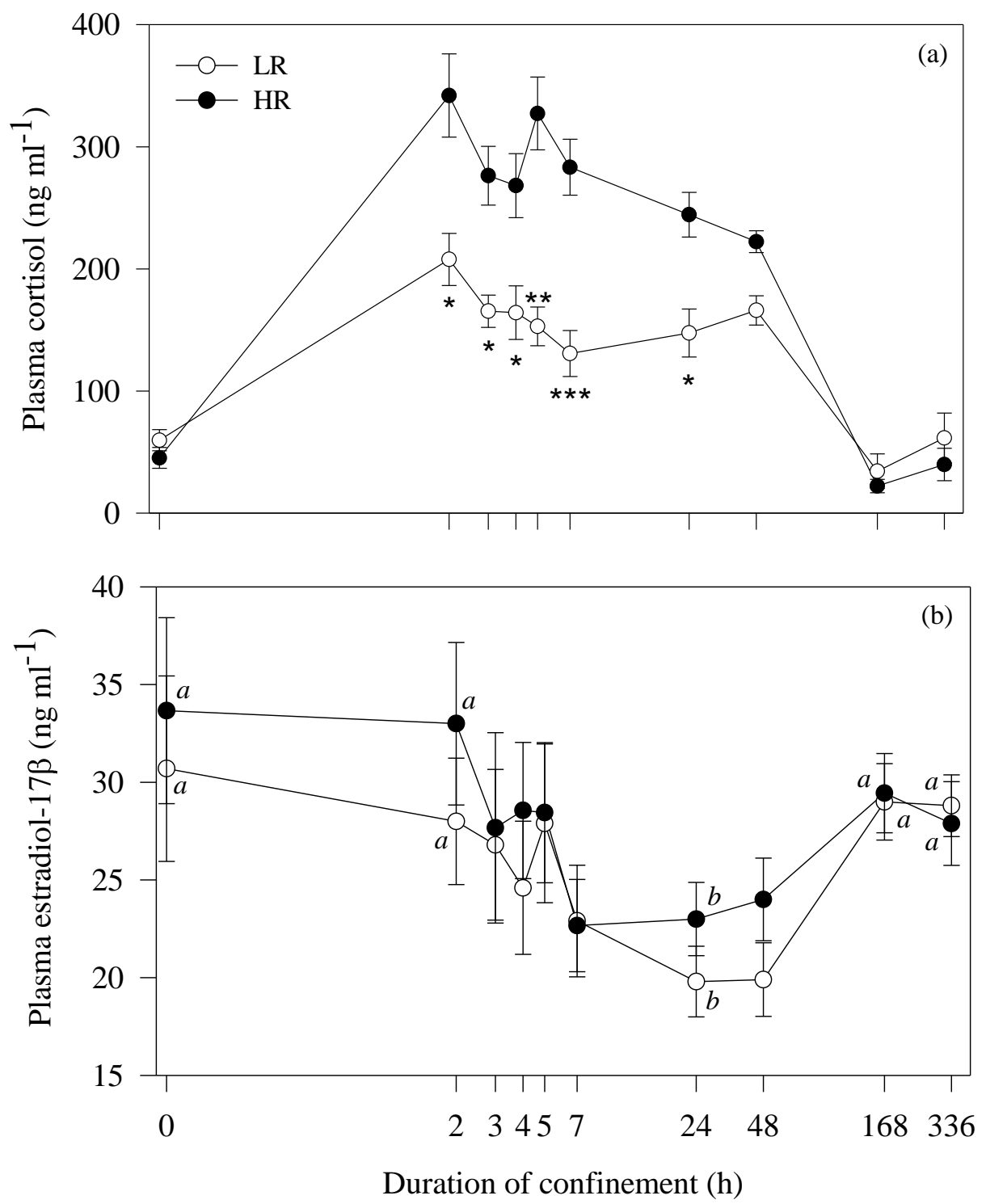

Figure 2 (a) Plasma cortisol and (b) plasma estradiol-17 $\beta$ levels in high- $(0)$ and low $(\bigcirc)$ responding female rainbow trout subjected to a 14 day period of confinement. Each point is the mean \pm SEM, $n=10$. Asterisks denote significant differences between HR and LR fish; $* P<0.05, * * P<0.01, * * * P<0.001$. Time points with the same letter are not significantly different from each other. $b$ denotes significantly different from $a, P<0.05$. 


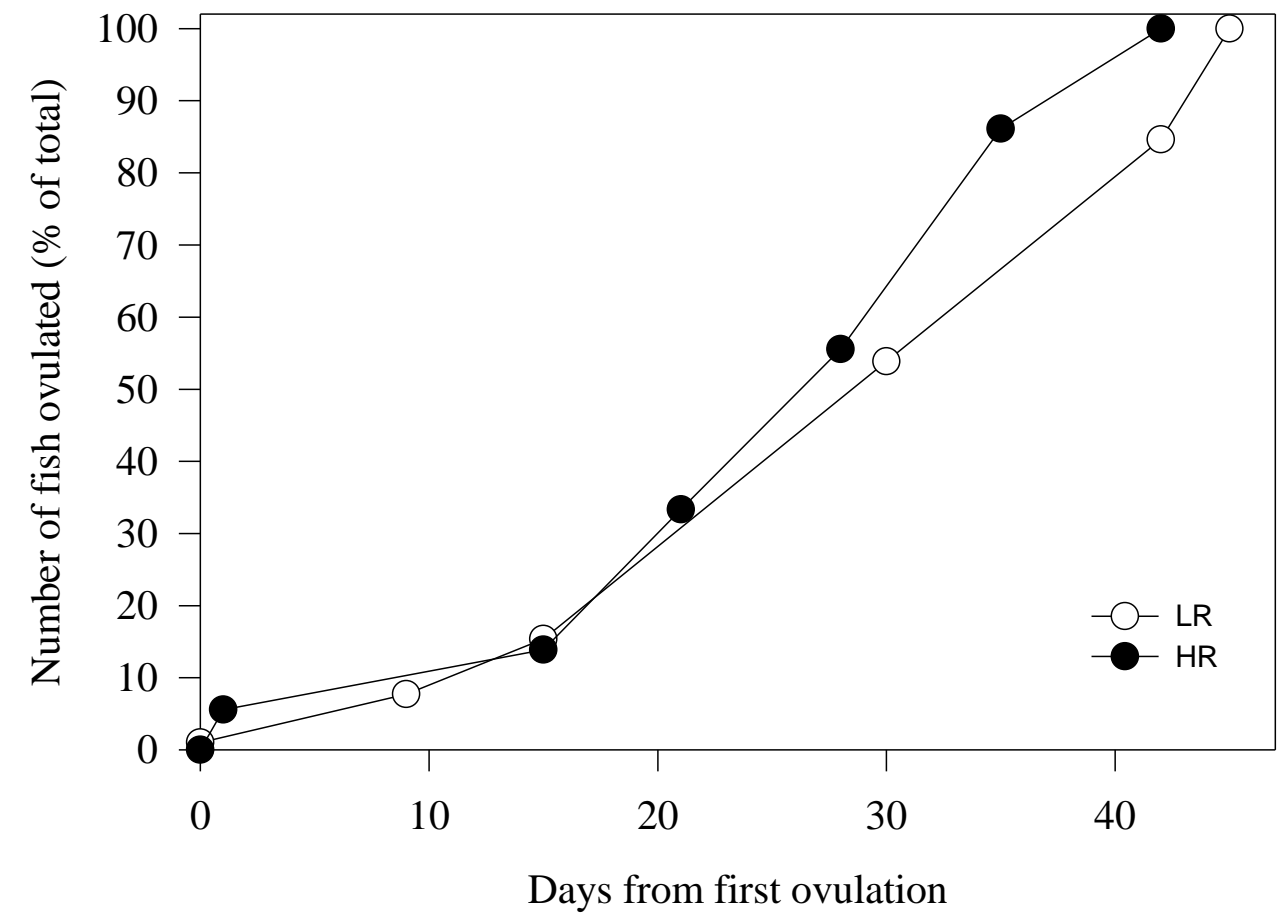

Figure 3 Rate of ovulation among mature female rainbow trout selected for highresponsiveness to stress $(\mathrm{HR} \bigcirc)$ and low-responsiveness to stress $(\mathrm{LR} O)$. $\mathrm{n}=14(\mathrm{LR}), 24$ (HR). 


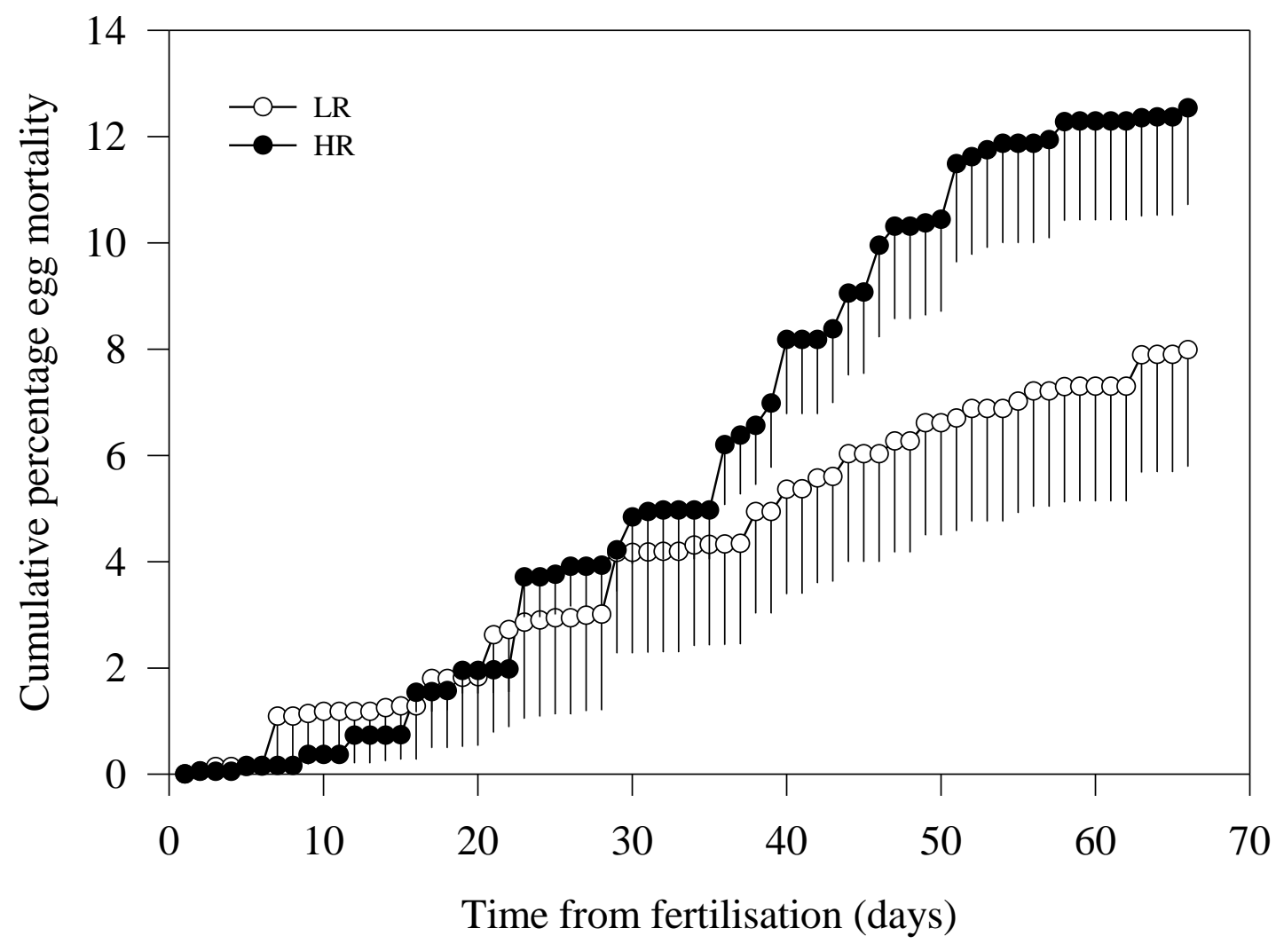

Figure 4 Mortality among batches of fertilized eggs produced by parents selected for highresponsiveness to stress (HR O) or low-responsiveness to stress $(\mathrm{LR} O)$. $\mathrm{n}=14(\mathrm{LR}), 24$ (HR). 